\title{
Purification of a polyclonal antibody against CD147 for ELISA using antigen-immunoaffinity chromatography
}

\author{
SHUANGSHUANG LIU, SHASHA LI, YANG ZHANG, YE WANG, YUMENG ZHU, BIN WANG and ZHI-NAN CHEN \\ Department of Cell Biology, National Translational Science Center for Molecular Medicine, \\ Fourth Military Medical University, Xi'an, Shaanxi 710032, P.R. China
}

Received February 23, 2016; Accepted February 20, 2017

DOI: $10.3892 / \mathrm{mmr} .2017 .6523$

\begin{abstract}
The immunoglobulin superfamily member CD147 is a widely expressed glycoprotein that occurs in both a membrane-spanning and soluble form. Sandwich ELISA is a powerful tool for analyzing soluble antigens. The aim of the present study was to obtain a highly specific polyclonal antibody against human CD147 that can be used for sandwich ELISA analysis. Expression of recombinant CD147 by a eukaryotic expression system was used to immunize rabbits to obtain antiserum. A highly specific polyclonal antibody that was able to detect soluble CD147 in sandwich ELISA was obtained by antigen-immunoaffinity chromatography purification. The purity of rabbit anti-CD147 polyclonal antibodies was $\sim 99 \%$, and ELISA analysis was able to determine the titer of the rabbit anti-CD147 polyclonal antibodies at 1:512,000. The lowest concentration of the standard CD147 antigen that the sandwich ELISA was able to detect was $31.25 \mathrm{pg} / \mathrm{ml}$. The sandwich ELISA system was composed of anti-hepatoma HAb18 monoclonal antibodies and purified rabbit anti-CD147 polyclonal antibodies. The present study demonstrated that antigen-immunoaffinity chromatography may be a good technique for the purification of polyclonal antibodies, which may be used to detect antigen in sandwich ELISAs.
\end{abstract}

\section{Introduction}

CD147, a member of the immunoglobulin (Ig) superfamily (1), is a transmembrane glycoprotein that is widely expressed in various cell types and at a high level in human tumors $(2,3)$; its expression has been reported to be upregulated in a number of cancer types $(4,5)$. The hepatoma-associated antigen HAb18G, which was cloned by anti-hepatoma monoclonal

Correspondence to: Dr Zhi-Nan Chen, Department of Cell Biology, National Translational Science Center for Molecular Medicine, Fourth Military Medical University, 17 Chang Le Western Road, Xi'an, Shaanxi 710032, P.R. China

E-mail: znchen@fmmu.edu.cn

Key words: soluble CD147, polyclonal antibody, purification, immunoaffinity chromatography, sandwich ELISA antibody (MAb) HAb18 screening of a human hepatocellular carcinoma cDNA library, has an identical nucleotide and amino acid sequence to CD147 $(6,7)$. Previous reports suggested that CD147 may be shed from the cell membrane via matrix metallopeptidase-dependent cleavage, which generates a soluble form of CD147 that may contain either one N-terminal Ig-like domain or two Ig-like domains $(8,9)$. Additional studies demonstrated that full-length CD147 may also be released via microvesicle shedding $(10,11)$. Soluble CD147 has also been indicated as a potential marker for the detection of certain types of cancer $(12,13)$.

ELISA is one of the basic applications of antibodies that is used to analyze soluble antigens (14); therefore, ELISA may be used to detect the concentration of soluble CD147 (15). We previously generated a murine antibody, HAb18, which targeted hepatocellular carcinoma-associated antigen HAb18G/CD147 (16). However, a successful sandwich ELISA detection system requires either MAbs that bind to independent sites on the antigen or affinity-purified polyclonal antibodies. Antibodies are widely used for the identification and localization of proteins due to their ability to bind an antigen with a high degree of affinity and specificity (17). MAbs have monospecificity, as they target a single epitope, which results in reduced cross-reaction (18). By contrast, polyclonal antibodies exhibit higher sensitivity, as a number of different epitopes are recognized (17). Owing to the various applications in which they may be used, antibodies with high specificity and sensitivity are desired. There are numerous methods used to purify antibodies, and the choice of purification procedure depends how the antibodies will be used and on the resources available (19). IgG may be purified by ammonium sulfate precipitation, ion-exchange chromatography, Protein A or Protein G affinity chromatography (20); occasionally, immunoaffinity chromatography is required to obtain more highly purified products (19). Currently, the majority of antibodies against CD147 are purified by Protein A or Protein G affinity chromatography. However, we have previously found that anti-CD147 polyclonal antibodies that are purified only by Protein A or Protein G affinity chromatography do not work well in the sandwich ELISAs to detect soluble CD147 (data not shown).

The present study produced a rabbit polyclonal antibody against HAb18G/CD147, which was purified by ammonium sulfate precipitation followed by antigen-immunoaffinity 
chromatography. This polyclonal antibody performed well with MAb HAb18 in the sandwich ELISA, which was used to detect soluble CD147.

\section{Materials and methods}

Preparation of eukaryotic-expressed CD147. Chinese hamster ovary (CHO)-derived cell line CHO-H8F8E10, that stably expresses HAb18GEP-Fc (a recombinant human protein containing the extracellular portion (EP) and the fragment crystallizable region $(\mathrm{Fc})$ of HAB18G/CD147, termed hereafter CD147-Fc), preserved in our laboratory, was cultured in SFM4 medium (Hyclone; GE Healthcare Life Sciences; Logan, UT, USA) at $37^{\circ} \mathrm{C}$. The recombinant eukaryotic expression vector pcDNA5/HAb18G-Fc, which contains the extracellular Ig-like domains of HAb18G with human Fc fragment in the C-terminal domain, was produced and large-scale cell culture was accomplished. Culture suspensions were collected and separated by tangential flow microfiltration (Sartorious AG, Göttingen, Germany). CD147-Fc recombinant protein was purified by Protein A chromatography with GE HiTrap rProtein A (GE Healthcare Life Sciences), according to the manufacturer's instructions. The Fc fragment was cleaved by Human Rhinovirus (HRV) 3C Protease (Sino Biological, Inc., Beijing, China). Briefly, CD147-Fc recombinant protein in cleavage buffer (Sino Biological, Inc.) was mixed with the recombinant HRV 3C protease in cleavage buffer at a mass ratio of 100:1 and incubated at $4^{\circ} \mathrm{C}$ overnight. The mixture was purified by $\mathrm{Ni}^{2+}$ affinity chromatography with GE HisTrap (GE Healthcare Life Sciences), followed by Protein A chromatography with GE HiTrap rProtein A, according to the manufacturer's instructions, in order to remove the residual $\mathrm{Fc}$ fragments and HRV 3C protease which also have a polyhistidine tag. Fractions were desalted and concentrated by an ultrafiltration device (Merck KGaA Darmstadt, Germany). The concentration of purified CD147 was determined by the Bicinchoninic Acid (BCA) assay (Thermo Fisher Scientific, Inc. Waltham, MA, USA). Samples were subsequently analyzed by $12 \%$ SDS-PAGE.

Immunization of rabbits with CD147. New Zealand white rabbits ( $\mathrm{n}=2$; weight 2.4 and $2.7 \mathrm{~kg}$; age, 7 months; housed at $12 \mathrm{~h} \mathrm{light/dark}$ cycles at $20-26^{\circ} \mathrm{C}$ and with free access to food and water) were used in the present study and handled in the Animal Center of the Fourth Military Medical University (Xi'an, China). All animal procedures were performed according to the University's Institutional Animal Care and Use Committee. The rabbits were given a hypodermic injection of recombinant purified CD147 protein $(800 \mu \mathrm{g})$, mixed with Complete Freund's Adjuvant (Sigma-Aldrich; Merck $\mathrm{KGaA}$ ) in a 1:1 ratio. After 3 weeks, the rabbits were boosted 2 times with CD147 $(400 \mu \mathrm{g})$ mixed with incomplete Freund's adjuvant (1:1) at 2-week intervals. Prior to each injection, blood samples were obtained from the marginal vein of the rabbit ear, centrifuged at $2,000 \mathrm{x}$ g for $10 \mathrm{~min}$ at $4^{\circ} \mathrm{C}$ and the sera was used to determine antibody titer by ELISA.

Antiserum titer determination by ELISA. The titer of antiserum was determined by indirect ELISA. For each well of the 96-well plate, $1 \mu \mathrm{g}$ of CD147 was diluted in $100 \mu \mathrm{l}$ of $0.1 \mathrm{M}$ sodium bicarbonate solution $\mathrm{pH} 9.6$ and incubated overnight at $4^{\circ} \mathrm{C}$. The plates were washed 3 times with PBS + Tween-20 (PBST; 0.05\% Tween-20), and then blocked with $1 \%$ bovine serum albumin (BSA; MP Biomedicals, LLC, Santa Ana, CA, USA) for $1 \mathrm{~h}$ at $37^{\circ} \mathrm{C}$. The plates were washed again 3 times with PBST and incubated with $100 \mu \mathrm{l}$ rabbit antisera against CD147 at 6 different dilutions between 1:16,000 and 1:512,000 for $1 \mathrm{~h}$ at $37^{\circ} \mathrm{C}$. Non-immune serum was used as a negative control. Following 3 washes with PBST, plates were incubated with $100 \mu 1$ diluted horseradish peroxidase (HRP)-conjugated goat anti-rabbit IgG (1:10,000 dilution; cat. no. 31460; Pierce; Thermo Fisher Scientific, Inc.) for $30 \mathrm{~min}$ at $37^{\circ} \mathrm{C}$. The reaction was developed by adding $100 \mu 1$ 3,3',5,5'-tetramethylbenzidine (TMB; Sigma-Aldrich; Merck KGaA) for $12 \mathrm{~min}$ at room temperature. Finally, $200 \mathrm{M}$ sulfuric acid was added to stop the reaction, and the absorbance was determined at $450 \mathrm{~nm}$ using a BioTeck Epoch microplate reader. The experiments were repeated two times.

Purification of rabbit anti-CD147 IgG. The polyclonal antibodies against CD147 were purified from the rabbit immune sera by ammonium sulfate precipitation followed by antigen-immunoaffinity chromatography. Briefly, rabbit blood was collected from the carotid artery following anesthesia with $40 \mathrm{mg} / \mathrm{kg}$ pentobarbital sodium and prior to sacrifice. Approximately $100 \mathrm{ml}$ blood was collected from each rabbit and $\sim 30 \mathrm{ml}$ immunized rabbit serum was collected by centrifugation at $1,000 \mathrm{x} \mathrm{g}$ for $10 \mathrm{~min}$ at $4^{\circ} \mathrm{C}$. Serum proteins were then precipitated in $50 \%$ ammonium sulfate at $4^{\circ} \mathrm{C}$ overnight and centrifuged at $2,000 \mathrm{xg}$ for $20 \mathrm{~min}$ at $4^{\circ} \mathrm{C}$. The pellet was subsequently dissolved in PBS (pH 7.4). To improve specificity, the polyclonal antibodies were purified by antigen-immunoaffinity chromatography (CNBr-activated sepharose 4B; GE Healthcare Life Sciences), according to the manufacturer's protocol. The purity and reactivity of the anti-CD147 polyclonal antibodies were analyzed by $10 \%$ SDS-PAGE [as described previously (21)] and indirect ELISA, respectively. The indirect ELISA was performed similar as the antiserum titer ELISA described above, with the purified polyclonal antibodies used in 6 serial dilutions from 40 to $0.625 \mathrm{ng} / \mathrm{ml}$.

Preparation of recombinant CD147 protein for antibody purification. As large amounts of coupling antigen are required for antigen-immunoaffinity chromatography, a prokaryotic expression system for CD147 was prepared. The extracellular domain of HAb18G/CD147 was amplified by polymerase chain reaction (PCR) using the expression plasmid pBluescript $\mathrm{KS}(+) / \mathrm{HAb} 18 \mathrm{G}$ as the template for the amplification. The primers used encompassed the entire transcript with SphI and MluI cloning sites added to the forward (CCCAAGCTT ATGGCGGCTGCGCTGTTCGTGCTG) and reverse (CGC GGATCCTCAGGA AGAGTTCCTGGCGGA) primers, respectively. PCR products were purified using the Wizard PCR preps kit (Promega Corporation, Madison, WI, USA). Following restriction endonuclease digestion (SphI and MluI; New England Biolabs, Inc., Ipswich, MA, USA), the fragment was inserted into the pGEX-6p-1 prokaryotic expression vector (GE Healthcare Life Sciences), which has a C-terminal glutathione S-transferase (GST) tag. Positive Escherichia coli BL21 (GE Healthcare Life Sciences) clones containing 
recombinant plasmid pGEX-6P-1/HAb18GEP were selected by growth on ampicillin-containing agar plates. A single colony of transformed $E$. coli was selected and cultured overnight at $37^{\circ} \mathrm{C}$ in Luria-Bertani (LB) medium (Thermo Fisher Scientific, Inc.) supplemented with $100 \mu \mathrm{g} / \mathrm{ml}$ ampicillin. The culture mixture was transferred to fresh LB medium (1:100 dilution) containing $100 \mu \mathrm{g} / \mathrm{ml}$ ampicillin and incubated at $37^{\circ} \mathrm{C}$ with continuous shaking until the absorbance at $600 \mathrm{~nm}$ reached 0.6-0.8. Expression conditions were optimized, and expression of the CD147-GST fusion proteins was induced by addition of $1 \mathrm{mM}$ isopropyl $\beta$-D-1-thiogalactopyranoside at $16^{\circ} \mathrm{C}$ for $5 \mathrm{~h}$. Following induction for $5 \mathrm{~h}$, the cells were harvested by centrifugation at $4,000 \mathrm{x}$ for $20 \mathrm{~min}$ at $4^{\circ} \mathrm{C}$. The supernatant was discarded and the pellet was resuspended in PBS (pH 7.4), and lysed by sonication on ice with 4-sec pulses at high intensity and a 7-sec cooling period between each burst for 120 cycles. The suspension was centrifuged at $12,000 \mathrm{x} \mathrm{g}$ for $50 \mathrm{~min}$ at $4^{\circ} \mathrm{C}$ to remove insoluble debris. The resultant supernatant was subsequently added to a GSTrap column (GE Healthcare Life Sciences) pre-equilibrated with PBS and the AKTA program was performed according to the GSTrap protocol. The flow-through was collected for SDS-PAGE analysis, as aforementioned, and the column was washed with PBS (pH 7.4). Finally, the bound protein was eluted with elution buffer (reduced glutathione; Amresco, LLC, Solon, OH, USA) and analyzed by $10 \%$ SDS-PAGE and western blot analysis. Fractions were desalted and concentrated by an ultrafiltration device with PBS (pH 7.4). The concentration of CD147-GST was determined by BCA assay.

SDS-PAGE and western blot analysis. Eukaryotic-expressed CD147 was separated by $12 \%$ non-reduced SDS-PAGE; prokaryotic-expressed CD147 and purified polyclonal antibodies were both separated by $10 \%$ non-reduced SDS-PAGE. All the samples were analyzed for protein concentration by BCA assay (Thermo Fisher Scientific, Inc.), and $10 \mu \mathrm{g}$ was loaded in each lane. Coomassie brilliant blue R250 (Sigma-Aldrich; Merck KGaA) was used to stain the gels. Quantification of gel staining was performed with GeneSnap software, version 4.0 (SynGene, Frederick, MD, USA). All the purification experiments described above were analyzed by coomassie brilliant blue staining, with the exception of the purified prokaryotic-expressed CD147 that was also analyzed by western blot analysis. Briefly, samples were transferred to a polyvinylidene fluoride microporous membrane (Merck KGaA) and probed with primary antibody HAb18 (22) overnight at $4^{\circ} \mathrm{C}$ and secondary horseradish peroxidase (HRP)-conjugated goat anti-mouse antibody (1:3,000 dilution; cat. no. A16072; Thermo Fisher Scientific, Inc.) for $50 \mathrm{~min}$ at room temperature. Signals were visualized by Western Blotting Detection Reagents (cat. no. 29100; Engreen Biosystem Co., Ltd., Beijing, China), using a Kodak 4000MM Image Station (Kodak; Rochester, NY, USA) and the Carestream Molecular Imaging Software, version 5.4.2 (Caresteam, Rochaster, NY, USA).

Establishment of sandwich ELISA. A monoclonal antibody against the extracellular domain of HAb18G/CD147, which was previously produced in our laboratory and designated HAb18 (22), was diluted in sodium bicarbonate solution ( $\mathrm{pH} 9.6)$ at the concentration of $10 \mu \mathrm{g} / \mathrm{ml}(100 \mu \mathrm{l} / \mathrm{well})$ and used to coat 96 -well plates. The plates were incubated at $4^{\circ} \mathrm{C}$ overnight, washed 3 times with PBST and blocked with $1 \%$ BSA $(200 \mu \mathrm{l} /$ well $)$ for $1 \mathrm{~h}$ at $37^{\circ} \mathrm{C}$. Subsequently, samples to be tested, or the highly purified CD147 protein (eukaryotic expression of the extracellular domain of HAb18G/CD147) were added to the individual wells and incubated at $37^{\circ} \mathrm{C}$ for $1 \mathrm{~h}$. Purified CD147 was used as a standard, serially diluted in $1 \%$ BSA/PBS $(1,000,500,250,125,62.5$, and $31.25 \mathrm{pg} / \mathrm{ml})$, of which $100 \mu \mathrm{l}$ was added to individual wells. After washing 3 times with PBST, anti-CD147 polyclonal antibodies, which were purified by different methods (including ammonium sulfate precipitation, antigen-immunoaffinity choromatography with ammonium sulfate precipitation and protein $\mathrm{A}$ chromatography with ammonium sulfate), or a commercial antibody against CD147 (cat. no. orb42082; Biorbyt Ltd., Cambridge, UK) were added (100 $\mu \mathrm{l} /$ well) and incubated at $37^{\circ} \mathrm{C}$ for $1 \mathrm{~h}$. After 3 washes with PBST, HRP-conjugated goat anti-rabbit IgG was added (100 $\mu \mathrm{l} /$ well) and the plate was incubated at $37^{\circ} \mathrm{C}$ for $30 \mathrm{~min}$. After washing 3 times with PBST, TMB substrate was added to the wells $(100 \mu \mathrm{l} /$ well $)$ and was measured at $450 \mathrm{~nm}$ using a BioTeck Epoch microplate reader. This test was repeated 15 times.

\section{Results}

Characterization of the CD147 antigen for immunization. As the first step in the production of the polyclonal antibodies against CD147, a CD147 protein was prepared. To obtain antibodies that resemble those produced in the human body, the eukaryotic-expressed CD147 was chosen as the immunogen. The recombinant expression vector pCDNA5/HAb18G-Fc contains extracellular domains of HAb18G and its C-terminal domain has an Fc fragment. The Fc fragment was cleaved by HRV 3C protease to avoid the production of antibodies against the Fc fragment. The Fc fragment and HRV 3C protease have polyhistidine tags, therefore, the mixture was purified by $\mathrm{Ni}^{2+}$ affinity chromatography followed by Protein A chromatography to obtain high-purity CD147 protein. The purified, eukaryotic-expressed CD147 was analyzed by 12\% SDS-PAGE under reducing conditions (Fig. 1). The recombinant human CD147-Fc protein consists of 422 amino acids and has a calculated molecular mass of $46.8 \mathrm{kDa}$; however, as a result of glycosylation, the recombinant protein migrates at $\sim 58-65 \mathrm{kDa}$ (Fig. 1, lane 1). The recombinant protein CD147-Fc has been degraded to some degree and exhibits dimer formation. The molecular mass of the cleaved extracellular domains of CD147 is $\sim 30-40 \mathrm{kDa}$ and the molecular mass of the HRV 3C protease is $\sim 21 \mathrm{kDa}$ (Fig. 1, lane 2). Following purification by $\mathrm{Ni}^{2+}$ affinity chromatography, a certain amount of recombinant CD147-Fc protein and HRV 3C protease remained (Fig. 1, lane 3). However, following Protein A chromatography, CD147 was of high purity and there were no residual $\mathrm{Fc}$ fragments or HRV 3C protease.

Antiserum titer analysis by ELISA. Rabbits were immunized with the purified eukaryotic CD147 immunogen, and the titer of the antiserum was detected by indirect ELISA. A total of 6 dilutions, between 1:16,000 and 1:512,000, of the antiserum were reacted with an equal amount of the 
recombinant $\mathrm{CD} 147$ protein. The antibody titer is defined as the highest dilution of serum at which the optical density 450 (OD450) ratio (OD450 of post-immunization serum/OD450 of pre-immunization serum) is $>2: 1$. The antibody titer was demonstrated to be $\sim 1: 512,000$ (Fig. 2). Generating a specific antibody preparation from low titer antiserum is difficult, and the production of a high-titer antiserum is a basic requirement for high-quality antibodies. The CD147 antiserum titer produced in the present study was high, which indicated that a strong response has been generated, and thus, the antibodies could be purified.

Characterization of the prokaryotic recombinant CD147 protein for antigen-immunoaffinity chromatography. Antigen-immunoaffinity chromatography requires large amounts of CD147 protein to purify the CD147 antibodies. Therefore, a prokaryotic expression system was used to make CD147 protein instead of the eukaryotic vector, as CD147 proteins may be expressed in large amounts in a short time and has increased stability compared with eukaryotic-expressed CD147. The extracellular domain sequence of the CD147 gene was cloned in an expression plasmid with a C-terminal GST tag, and the construct was transformed into E. coli BL21 competent cells. The GST tag was used to purify the recombinant CD147 with a GSTrap affinity column. The purified CD147-GST fusion protein, whose expected size is $\sim 44 \mathrm{kDa}$, was analyzed by $10 \%$ SDS-PAGE and confirmed by western blot analysis with anti-CD147 MAb HAb18 (Fig. 3). SDS-PAGE and western blot analysis demonstrated that purification of CD147 was of high purity and confirmed the presence of a corresponding band for expressed CD147-GST protein. This high-quality purified CD147 protein is required to produce high-quality purified antibodies.

Assessment of the purity and reactivity of the anti-CD147 polyclonal antibody by SDS-PAGE and indirect ELISA. A non-reducing SDS-PAGE was used to determine the purity of rabbit anti-CD147 IgG (120-150 kDa), which was purified by ammonium sulfate precipitation followed by antigen-immunoaffinity chromatography (Fig. 4A, lane 1). Compared with the antiserum (Fig. 4A, lane 5) and the antibodies purified only by ammonium sulfate precipitation (Fig. 4A, lane 4), the affinity-purified antibodies had a higher purity; the purity of the rabbit anti-CD147 IgG was $\sim 99 \%$.

To determine the reactivity of the purified antibodies, an indirect ELISA was performed. Antibodies were purified by ammonium sulfate precipitation followed by antigen-immunoaffinity chromatography, and different dilutions $(0.625-40 \mathrm{ng} / \mathrm{ml})$ were reacted with an equal amount of CD147 protein. The result demonstrated in Fig. 4B indicated that the purified antibody had a good affinity for CD147, which indicated that the purified antibody has a high reactivity.

Establishment of sandwich ELISA. A sandwich ELISA was constructed with the following components: i) The HAb18 anti-CD147 MAb,previously produced in our laboratory (22), was used as the capture antibody; ii) the highly purified eukaryotic CD147 was used as a standard protein, which was serially diluted to $1000,500,250,125,62.5,31.25 \mathrm{pg} / \mathrm{ml}$ and iii) the anti-CD147 polyclonal antibodies were used as the detecting
Table I. Sensitivity test results of human CD147 sandwich ELISA.

\begin{tabular}{lc}
$\begin{array}{l}\text { CD147 standard concentration } \\
(\mathrm{pg} / \mathrm{ml})\end{array}$ & Average OD $450^{\mathrm{a}} \pm \mathrm{SD}$ \\
\hline 1,000 & $1.765 \pm 0.021$ \\
500 & $0.884 \pm 0.005$ \\
250 & $0.587 \pm 0.029$ \\
125 & $0.321 \pm 0.020$ \\
62.5 & $0.193 \pm 0.005$ \\
31.25 & $0.164 \pm 0.009$ \\
Blank & $0.069 \pm 0.007$ \\
\hline
\end{tabular}

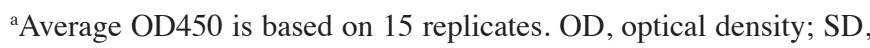
standard deviation.

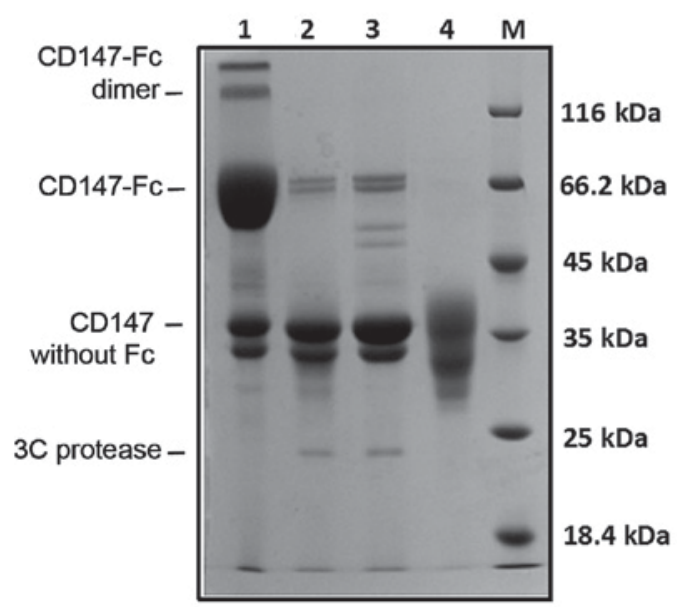

Figure 1. SDS-PAGE analysis of the CD147 antigen prior to rabbit immunization. Eukaryotic-expressed recombinant protein CD147-Fc was treated with HRV 3C protease for $16 \mathrm{~h}$, and the mixture was purified by $\mathrm{Ni}^{2+}$ affinity chromatography followed by Protein A chromatography. SDS-PAGE analysis demonstrated that CD147 was of high purity and indicated that there was no residual $\mathrm{Fc}$ fragment or $3 \mathrm{C}$ protease. Lane 1, recombinant eukaryotic-expressed CD147-Fc, which has a dimer formation and has been partially degraded; Lane 2, the recombinant CD147-Fc protein was treated with HRV 3C protease for $16 \mathrm{~h}$, resulting in a mixture of CD147-Fc, CD147, Fc fragments and HRV 3 C protease; Lane 3, mixture purified by $\mathrm{Ni}^{2+}$ affinity chromatography, which is not very powerful, and a certain amount of recombinant protein and HRV 3C protease remain; Lane 4, CD147 immunogen purified by Protein A chromatography, no recombinant protein or HRV $3 \mathrm{C}$ protease was detected; Lane M, protein molecular weight marker. HRV, human rhinovirus.

antibody. Detection of the standard proteins was performed under optimized conditions. The present study demonstrated that the sandwich ELISA system detected the concentration of $\mathrm{CD} 147$ as low as $31.25 \mathrm{pg} / \mathrm{ml}$ (Table I). By contrast, the present study established other sandwich ELISA systems using various rabbit anti-CD147 polyclonal antibodies that were purified by methods other than antigen-immunoaffinity chromatography. The results demonstrated that only the system comprised of antibodies purified by antigen-immunoaffinity chromatography was able to detect CD147 protein at concentrations as low as $31.25 \mathrm{pg} / \mathrm{ml}$ (Table II). 
Table II. Comparison of polyclonal antibodies against CD147 purified by antigen-immunoaffinity chromatography and other methods of purification.

Source

Present study

Present study

Present study

Purchased from Biorbyt

Ltd., Cambridge, UK
Background of sandwich ELISA (OD450)
Lowest concentration of CD147 detected (pg/ml)

All methods of purification included a first step of ammonium sulfate precipitation, prior to chromatography. OD, optical density.

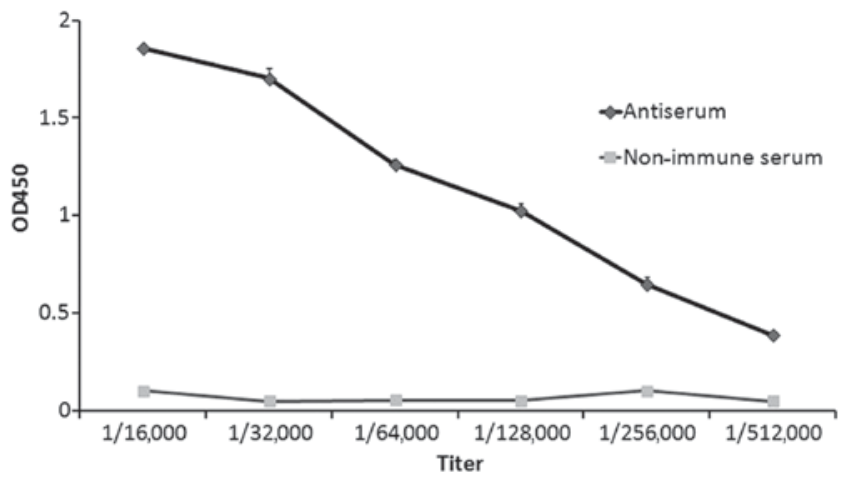

Figure 2. Analysis of antiserum titer by ELISA. The antibody titer is defined as the highest dilution of serum at which the OD450 ratio (OD450 of post-immunization serum/OD450 of pre-immunization serum) is $>2: 1$. For non-immune serum at 1:512,000, the OD450 is 0.049 , and for antiserum at $1: 512,000$, the OD450 is 0.387 ; therefore, the ratio is $>2: 1$, and the titer of this antiserum is $\sim 1: 512,000$. Data are presented as the mean + standard deviation. OD, optical density.

A

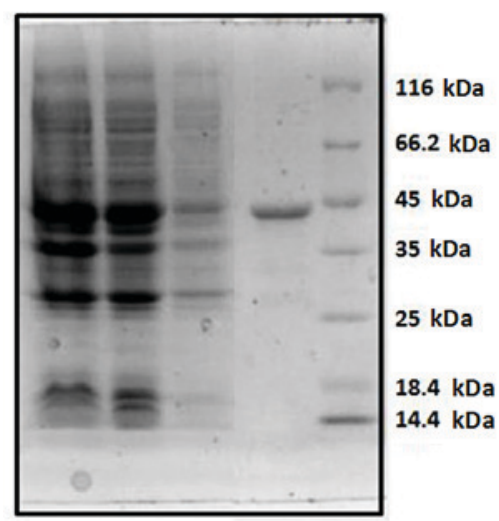

B

Figure 3. SDS-PAGE and western blot analysis of CD147-GST fusion protein purification and identification. The prokaryotic-expressed CD147-GST fusion protein was purified by a GSTrap column. The purified protein had a high purity. (A) SDS-PAGE analysis of purified CD147-GST. Lane 1, supernatant of the lysate of CD147-GST; Lane 2, flow through liquid of the supernatant; Lane 3, flow through liquid of the washing buffer; Lane 4, protein eluted from the column; Lane M, protein molecular weight marker. (B) Western blot analysis of purified CD147-GST. The primary antibody was HAb18, which has high specificity and affinity for CD147. The secondary antibody was goat anti-mouse immunoglobulin $\mathrm{G}$ conjugated with horseradish peroxidase. Western blotting confirmed that the observed band corresponded to CD147-GST protein. GST, glutathione S-transferase.
A

$\begin{array}{lc}0.069 & 31.25 \\ 0.17 & 250 \\ 0.13 & 250 \\ 0.08 & 125\end{array}$

.25 25 
was high enough to produce high quality antibodies. The purification of immunoglobulins presents certain practical complications, particularly for polyclonal antibodies (24). There are various types of methods for the purification of antibodies (4); however, the choice of the purification method depends on the application of the antibodies (3). In the present study, polyclonal antibodies against CD147 were produced for sandwich ELISA to detect soluble CD147 antigen in serum; therefore, antigen-immunoaffinity chromatography was used to purify polyclonal antibodies to obtain highly specificity anti-CD147 antibodies. Immunoaffinity chromatography uses biologically associated binding agents and is used to selectively purify or analyze a target compound (25). In the current study, polyclonal antibodies against CD147 were purified by antigen-immunoaffinity chromatography following ammonium sulfate precipitation. The conditions of the antigen-immunoaffinity chromatography were optimized, including the buffer type and $\mathrm{pH}$, and particularly the concentration of the reactors and reaction time $(25,26)$. Following purification, antibodies with a purity of $\sim 99 \%$ were obtained. In an indirect ELISA against CD147, the reactivity of the polyclonal antibodies purified by antigen-immunoaffinity chromatography was demonstrated to be high. The polyclonal antibody against CD147 and the MAb HAb18 made up the basic system of the sandwich ELISA for detecting soluble CD147. The lower limit of the ELISA method used for detecting the CD147 standard was $31.25 \mathrm{pg} / \mathrm{ml}$. By contrast, with antibodies purified by Protein A or ammonium sulfate precipitation, the sensitivity of the ELISA using antibodies purified by antigen-immunoaffinity chromatography was the highest.

The polyclonal antibody purified by antigen-immunoaffinity chromatography may be a novel tool for further investigation of soluble CD147 in human serum. The sandwich ELISA kit described in the present study, which includes the antigen-immunoaffinity chromatography purified polyclonal antibody, may be used to detect the presence of CD147 in several types of cancers to investigate whether soluble CD147 is a biomarker in certain cancers. Meanwhile, the purification method discussed in the present study may be applied to the purification of various other antibodies.

\section{Acknowledgements}

The present study was supported by grants from the National Science and Technology Major Project (grant no. 2012AA02A301).

\section{References}

1. Biswas C: Tumor cell stimulation of collagenase production by fibroblasts. Biochem Biophys Res Commun 109: 1026-1034. 1982.

2. Ellis SM, Nabeshima K and Biswas C: Monoclonal antibody preparation and purification of a tumor cell collagenase-stimulatory factor. Cancer Res 49: 3385-3391, 1989.

3. Polette M, Gilles C, Marchand V, Lorenzato M, Toole B, Tournier JM, Zucker S and Birembaut P: Tumor collagenase stimulatory factor (TCSF) expression and localization in human lung and breast cancers. J Histochem Cytochem 45: 703-709, 1997.

4. Tang J, Wu YM, Zhao P, Yang XM, Jiang JL and Chen ZN: Overexpression of HAb18G/CD147 promotes invasion and metastasis via alpha3beta1 integrin mediated FAK-paxillin and FAK-PI3K-Ca2+ pathways. Cell Mol Life Sci 65: 2933-2942, 2008.
5. Wu Y, Zhou X and Zheng PS: Involvement of CD147 isoform-4 in the proliferation of $\mathrm{SiHa}$ cells: A possible molecular mechanism of cervical cancer. Oncol Rep 26: 717-724, 2011.

6. Chen ZN, Yang Z and Mi L: Analysis on the structure and function of hepatoma transfer-associated factor HAb18G (in Chinese) J Mol Cell Immunol 15: 34, 1999.

7. Jiang JL, Zhou Q, Yu MK, Ho LS, Chen ZN and Chan HC: The involvement of $\mathrm{HAb} 18 \mathrm{G} / \mathrm{CD} 147$ in regulation of store-operated calcium entry and metastasis of human hepatoma cells. J Biol Chem 276: 46870-46877, 2001.

8. Haug C, Lenz C, Diaz F and Bachem MG: Oxidized low-density lipoproteins stimulate extracellular matrix metalloproteinase Inducer (EMMPRIN) release by coronary smooth muscle cells. Arterioscler Thromb Vasc Biol 24: 1823-1829, 2004

9. Egawa N, Koshikawa N, Tomari T, Nabeshima K, Isobe T and Seiki M: Membrane type 1 matrix metalloproteinase (MT1-MMP/MMP-14) cleaves and releases a 22-kDa extracellular matrix metalloproteinase inducer (EMMPRIN) fragment from tumor cells. J Biol Chem 281: 37576-37585, 2006.

10. Tang Y, Kesavan P, Nakada MT and Yan L: Tumor-stroma interaction: Positive feedback regulation of extracellular matrix metalloproteinase inducer (EMMPRIN) expression and matrix metalloproteinase-dependent generation of soluble EMMPRIN. Mol Cancer Res 2: 73-80, 2004.

11. Taylor PM, Woodfield RJ, Hodgkin MN, Pettitt TR, Martin A, Kerr DJ and Wakelam MJ: Breast cancer cell-derived EMMPRIN stimulates fibroblast MMP2 release through a phospholipase $\mathrm{A}(2)$ and 5-lipoxygenase catalyzed pathway. Oncogene 21: 5765-5772, 2002

12. Yanaba K, Asano Y, Tada Y, Sugaya M, Kadono T, Hamaguchi Y and Sato S: Increased serum soluble CD147 levels in patients with systemic sclerosis: Association with scleroderma renal crisis. Clin Rheumatol 31: 835-839, 2012.

13. Wu J, Hao ZW, Zhao YX, Yang XM, Tang H, Zhang X, Song F, Sun XX, Wang B, Nan G, et al: Full-length soluble CD147 promotes MMP-2 expression and is a potential serological marker in detection of hepatocellular carcinoma. J Transl Med 12: 190, 2014

14. Hornbeck PV: Enzyme-linked immunosorbent assays. Curr Protoc Immunol 110: 1-23, 2015.

15. Moonsom S, Tayapiwatana C, Wongkham S, Kongtawelert $P$ and Kasinrerk W: A competitive ELISA for quantifying serum CD147: Reduction of soluble CD147 levels in cancer patient sera. Hybridoma (Larchmt) 29: 45-52, 2010.

16. Ku XM, Liao CG, Li Y, Yang XM, Yang B, Yao XY, Wang L, Kong LM, Zhao $\mathrm{P}$ and Chen ZN: Epitope mapping of series of monoclonal antibodies against the hepatocellular carcinoma-associated antigen HAb18G/CD147. Scand J Immunol 65: 435-443, 2007.

17. Nelson PN, Westwood OM, Jefferis R, Goodall M and Hay FC: Characterisation of anti-IgG monoclonal antibody $\mathrm{A} 57 \mathrm{H}$ by epitope mapping. Biochem Soc Trans 25: 373S, 1997.

18. Lipman NS, Jackson LR, Trudel LJ and Weis-Garcia F: Monoclonal versus polyclonal antibodies: Distinguishing characteristics, applications, and information resources. ILAR J 46: 258-268, 2005.

19. Lane D: Antibodies: A Laboratory Manual. Cold Spring Harbor Press, Cold Spring Harbor, N.Y, pp288-312, 1998.

20. Andrew SM and Titus JA: Purification of immunoglobulin G. Curr Protoc Immunol: Chapter 2: Unit 2.7, 2001.

21. Laemmli UK: Cleavage of structural proteins during the assembly of the head of bacteriophage T4. Nature 227: 680-685, 1970.

22. Chen ZN and Liu YF: Monoclonal antibody HAb18 to human hepatoma. J Monocl Antibod 3: 15-17, 1990 (In Chinese).

23. Howard GC and Bethell DR: Basic methods in antibody production and characterization. CRC Press Ltd, Boca Raton, Florida, pp1-49, 2000

24. Verdoliva A, Basile G and Fassina G: Affinity purification of immunoglobulins from chicken egg yolk using a new synthetic ligand. J Chromatogr B Biomed Sci Appl 749: 233-242. 2000.

25. Hage DS: Survey of recent advances in analytical applications of immunoaffinity chromatography. J Chromatogr B Biomed Sci Appl 715: 3-28. 1998.

26. Hage DS and Phillips TM: Immunoaffinity chromatography. In: Taylor \& Francis, Handbook of Affinity Chromatography. New York, Chapter 6, 2006. 